Control

Elsevier Editorial system(tm) for Food

Manuscript Draft

Manuscript Number: FOODCONT-D-19-03127

Title: Exploring the potential of NIR hyperspectral imaging for automated quantification of rind amount in grated Parmigiano Reggiano cheese

Article Type: Research Paper

Keywords: Grated cheese; Rind percentage; NIR hyperspectral imaging; Multivariate calibration; Multivariate image analysis

Corresponding Author: Professor Alessandro Ulrici,

Corresponding Author's Institution: Università di Modena e Reggio Emilia

First Author: Rosalba Calvini

Order of Authors: Rosalba Calvini; Sara Michelini; Valentina Pizzamiglio; Giorgia Foca; Alessandro Ulrici

Abstract: Parmigiano Reggiano (P-R) is one of the most important Italian food products labelled with Protected Designation of Origin (PDO). The PDO denomination is applied also to grated $\mathrm{P}-\mathrm{R}$ cheese products meeting the requirements regulated by the Specifications of Parmigiano Reggiano Cheese. Different quality parameters are monitored, including also the percentage of rind, which is edible and should not exceed the limit of 18\% $(\mathrm{w} / \mathrm{w})$. The present study aims at evaluating the possibility of using near infrared hyperspectral imaging (NIR-HSI) to quantify the rind percentage in grated Parmigiano Reggiano cheese samples in a fast and non-destructive manner. Indeed, NIR-HSI allows the simultaneous acquisition of both spatial and spectral information from a sample, resulting more suitable than classical single-point spectroscopy for the analysis of heterogeneous samples like grated cheese. Hyperspectral images of grated $\mathrm{P}-\mathrm{R}$ cheese samples containing increasing levels of rind were acquired in the 900-1700 nm spectral range. Each hyperspectral image was firstly converted into a one-dimensional signal, named

hyperspectrogram, which codifies the relevant information contained in the image. Then, the matrix of hyperspectrograms was used to calculate a calibration model for the prediction of the rind percentage using Partial Least Squares (PLS) regression. The calibration model was validated considering two external test sets of samples, confirming the effectiveness of the proposed approach.

Suggested Reviewers: Jose Manuel Amigo PhD

Professor, Analytical Chemistry, Universidad del País Vasco

josemanuel. amigo@ehu. eus

Prof. Amigo is expert in Chemometrics and in Hyperspectral Imaging; many of his research works are focused on the application of hyperspectral imaging for the characterization of food samples.

Paolo Oliveri PhD

University researcher, Department of Pharmacy, University of Genova oliveri@difar.unige.it 
Dr. Oliveri is expert in the use of chemometrics for the characterization of food samples.

Paul Williams PhD

University researcher, Deparment of Food Science, Stellenbosch University pauljw@sun.ac.za

Dr. Williams does research in Vibrational Spectroscopy, Hyperspectral Imaging and Multivariate Data Analysis. 
Dipartimento di Scienze della Vita

Reggio Emilia, October 31, 2019

Dear Editor,

please find enclosed a copy of the manuscript:

Rosalba Calvini, Sara Michelini, Valentina Pizzamiglio, Giorgia Foca, Alessandro Ulrici

Exploring the potential of NIR hyperspectral imaging for automated quantification of rind amount in grated Parmigiano Reggiano cheese

that we would like to be considered for publication in Food Control.

This article describes the development of a fast and non-destructive method based on near infrared hyperspectral imaging (NIR-HSI) for the quantification of rind percentage in grated Parmigiano Reggiano cheese. Although edible, in long-ripened cheeses such as Parmigiano Reggiano the rind has chemical and textural properties different from those of the inner part of the cheese. As a consequence, an excessive amount of rind in grated cheese can be perceived by consumers, negatively affecting the organoleptic properties of the product. For this reason, the percentage of rind in grated P-R cheese should not exceed the $18 \%(w / w)$ threshold value.

Since NIR-HSI allows to obtain both spatial and spectral information from a sample by detecting variations in the chemical composition over the sample surface, it can be effectively applied to the analysis of grated cheese, which is an heterogeneous food matrix composed by unevenly dispersed particles derived from both cheese rind and pulp.

In this work, 45 grated cheese samples containing different percentages of rind (from $0 \%$ to $40 \%$ ) were considered. NIR hyperspectral images were acquired in the 900-1700 nm spectral range on three different aliquots of each sample, for a total of 135 images. In order to develop calibration models, the images were converted into a matrix of signals, named hyperspectrograms, which were obtained by merging in sequence the frequency distribution curves of quantities derived from a PCA model common to the whole dataset of images. The matrix of hyperspectrograms was then analysed by means of Partial Least Squares (PLS) algorithm for the determination of the amount of rind. The PLS calibration model was validated considering two external test sets of samples, confirming the effectiveness of the proposed approach. These results demonstrated the possibility to develop a fast and non-destructive method based on NIR-HSI to control the amount of rind in grated Parmigiano Reggiano cheese samples.

The article is original, unpublished and not being considered for publication elsewhere.

Best regards,

Alessandro Ulrici

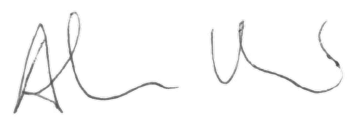

Prof. Alessandro Ulrici

Dipartimento di Scienze della Vita

Università degli Studi di Modena e Reggio Emilia

Padiglione Besta - Via Amendola, 2 - 42122 Reggio Emilia - Italy

Phone +390522522043

Fax +39 0522522027

email: alessandro.ulrici@unimore.it 


\title{
Exploring the potential of NIR hyperspectral imaging for automated quantification of rind amount in grated Parmigiano Reggiano cheese
}

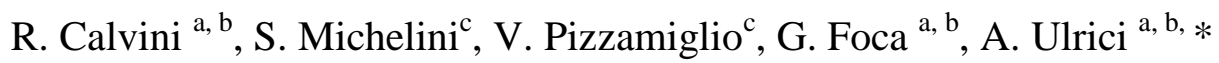 \\ ${ }^{a}$ Department of Life Sciences, University of Modena and Reggio Emilia, Pad. Besta, Via Amendola, \\ 2, Reggio Emilia, 42122, Italy \\ b Interdepartmental Centre BIOGEST-SITEIA, University of Modena and Reggio Emilia, Piazzale \\ Europa, 1, Reggio Emilia, 42122, Italy \\ ${ }^{c}$ Parmigiano Reggiano Cheese Consortium, Via J.F. Kennedy, 18, Reggio Emilia, 42124, Italy \\ * Corresponding author: alessandro.ulrici@unimore.it
}

\begin{abstract}
Parmigiano Reggiano (P-R) is one of the most important Italian food products labelled with Protected Designation of Origin (PDO). The PDO denomination is applied also to grated P-R cheese products meeting the requirements regulated by the Specifications of Parmigiano Reggiano Cheese. Different quality parameters are monitored, including also the percentage of rind, which is edible and should not exceed the limit of $18 \%(\mathrm{w} / \mathrm{w})$. The present study aims at evaluating the possibility of using near infrared hyperspectral imaging (NIR-HSI) to quantify the rind percentage in grated Parmigiano Reggiano cheese samples in a fast and non-destructive manner. Indeed, NIRHSI allows the simultaneous acquisition of both spatial and spectral information from a sample, resulting more suitable than classical single-point spectroscopy for the analysis of heterogeneous samples like grated cheese. Hyperspectral images of grated P-R cheese samples containing increasing levels of rind were acquired in the 900-1700 nm spectral range. Each hyperspectral image was firstly converted into a one-dimensional signal, named hyperspectrogram, which codifies the relevant information contained in the image. Then, the matrix of hyperspectrograms was used to calculate a calibration model for the prediction of the rind percentage using Partial Least Squares (PLS) regression. The calibration model was validated considering two external test sets of samples, confirming the effectiveness of the proposed approach.
\end{abstract}

\section{Keywords}

Grated cheese; Rind percentage; NIR hyperspectral imaging; Multivariate calibration; Multivariate image analysis. 


\section{Introduction}

Parmigiano Reggiano (P-R) is a long-ripened, cooked, hard cheese produced in Italy and registered with Protected Denomination of Origin (PDO). P-R represents one of the most important typical Italian food products and it is exported worldwide. This cheese is manufactured from raw and unheated bovine milk, and the whole production chain must take place in a restricted area in Northern Italy (Malacarne et al., 2008).

The PDO is extended also to grated cheese obtained from Parmigiano Reggiano cheese wheels, provided that the product is grated in the specific production area and packaged immediately afterwards, in order to avoid modifications of its organoleptic properties.

Furthermore, grated cheese products designated as Parmigiano Reggiano should meet technical and technological parameters ruled by the Specifications of Parmigiano Reggiano Cheese (https://www.parmigianoreggiano.com/consortium/rules_regulation_2/default.aspx ), that regulates all stages of P-R production, including cow feeding, cheese manufacturing and ripening process.

One of the different quality parameters of grated P-R cheese regulated by the Specifications is the amount of rind. The rind is the external part of cheese wheels; although edible, in long-ripened cheeses it has chemical and physical properties different from those of the inner part of the cheese. These differences are mainly caused by exposure to environmental conditions during ripening, which determines a decrease in moisture content, proteolytic activity and a higher degree of oxidation (Cattaneo et al., 2008; Karoui et al., 2007; Malacarne et al., 2019). Furthermore, the different properties of rind and pulp also affect size and shape of grated particles. In fact, generally rind particles are finer and less circular than those derived from the pulp (Alinovi et al., 2019). As a consequence of its peculiar chemical and textural properties, an excessive amount of rind in grated cheese can be perceived by consumers, negatively affecting the organoleptic properties of the product (Zannoni and Hunter, 2015). For these reasons, the percentage of rind in grated P-R cheese should not exceed the $18 \%(\mathrm{w} / \mathrm{w})$ threshold value.

In order to ensure quality compliance of commercial products of grated P-R cheese and to avoid counterfeits, it is essential to implement effective analytical methods to correctly estimate the rind amount, meeting the requirements of low costs, limited sample preparation and short times of analysis. In this context, Near Infrared (NIR) spectroscopy has been widely employed for fast and non-destructive analysis and characterization of food products, thanks to its ability to easily provide a spectral fingerprint codifying the chemical composition of the analysed sample (Curda et al., 2004; Woodcok et al., 2008; Foca et al., 2013; Kraggerud et al.; 2014). 
In particular, previous research studies evaluated the effectiveness of NIR spectroscopy in verifying the authenticity of P-R grated cheese and in discriminating compliant from non-compliant samples (Cevoli et al., 2013a, Cevoli et al., 2013b).

However, grated cheese is an inhomogeneous food matrix composed by unevenly dispersed particles derived from both cheese rind and pulp, which are characterized by different chemical properties. When dealing with heterogeneous samples, classical NIR spectroscopy may lead to inaccurate results since it is based on the acquisition of "average" spectra over a given sample area, thus losing the information related to the compositional variability within the sample. The importance of spatial information for the analysis of P-R grated cheese was recently demonstrated by Alinovi et al. (2019), which found a relationship between rind percentage and parameters related to particle size and distribution calculated from digital RGB images of the cheese samples.

The advantages of image-based methods and NIR spectroscopy can be coupled in NIR Hyperspectral Imaging (NIR-HSI), an analytical technique based on the acquisition of particular types of images, called hyperspectral images, where a whole NIR spectrum is registered for each image pixel (Gowen et al., 2007; Amigo et al., 2013; Calvini et al.; 2018). More in detail, a hyperspectral image, also called hypercube, is a three-dimensional data array with two spatial dimensions ( $x$ pixel rows and $y$ pixel columns) and one spectral dimension, corresponding to the $\lambda$ acquired wavelengths. Therefore, the hypercube can be seen as a stack of spectrally resolved images, each one acquired at a given wavelength, or as a series of spatially resolved spectra, where each spectrum characterizes one pixel of the image (Wu and Sun, 2013a; Amigo et al., 2015; Calvini et al.; 2016).

Considering that each NIR spectrum acts like a fingerprint of the chemical properties of a specific pixel, thanks to hyperspectral imaging it is possible to obtain both spatial and spectral information from a sample by observing variations in the chemical composition over the sample surface. These aspects resulted to be particularly useful for the analysis of heterogeneous matrices like food products (Wu and Sun, 2013b; Dale et al., 2013; Liu et al.,2017), generally overcoming the performances obtained with single-point spectroscopy (Burger and Geladi, 2006; Shonbichler et al., 2013).

However, the high amount of information contained in hyperspectral images can also become a drawback, since each hypercube can contain up to tens of thousands of pixel spectra, resulting in data handling and data storage issues. Indeed, in order to extract the relevant information from this kind of data, the application of proper multivariate statistical methods is mandatory (Burger and Gowen, 2011). This is known as Multivariate Image Analysis (MIA), which is based on the application to images of common chemometric methods, like e.g. Principal Component Analysis 
(PCA). This approach essentially consists in considering each pixel of the image as a separate object and the main goal is to find similarities or differences among clusters of pixels based on their spectral signatures (Prats-Montalban et al., 2011; Amigo et al., 2015).

When dealing with a large number of hyperspectral images that should be analysed altogether, classical pixel-level MIA can become unfeasible due to the intensive computational loads, since it would imply the simultaneous analysis of numerous images, each one with tens of thousands of pixel spectra. In these situations, a possible solution is to move from a pixel-level approach to an image-level approach, which consists in performing the analysis considering the image of each sample as a single object and extracting a feature vector characterizing the whole image, and thus the corresponding sample. In this manner, it is possible to analyse data matrices containing these feature vectors, in order to gain a general overview of the image dataset, to identify images sharing similar features or to quantify whole sample properties (Ulrici et al., 2012; Kucheryavskiy, 2013; Giraudo et al., 2018; Orlandi et al., 2018a; Orlandi et al., 2018b; Oliveri et al., 2019).

To this aim, a data dimensionality reduction method has been proposed, which consists in converting each hyperspectral image of the dataset into a one-dimensional signal, named hyperspectrogram, obtained by merging in sequence the frequency distribution curves of quantities derived from a PCA model calculated on the images (Ferrari et al., 2013; Ferrari et al., 2015; Xu et al., 2016; Calvini et al., 2016; Corti et al., 2017). In this manner, each hyperspectrogram summarizes the relevant information contained in the corresponding hyperspectral image and a large dataset of hyperspectral images is converted into a matrix of signals, which in turn can be analysed by means of common chemometric methods.

In this context, the main goal of the present study consisted in evaluating the possibility of exploiting the advantages of NIR-HSI coupled to data dimensionality reduction, in order to monitor the rind percentage of grated P-R cheese products. In particular, hyperspectral images of grated P-R cheese samples were analysed by means of the hyperspectrograms approach, and the resulting hyperspectrograms were then used to predict the rind percentage by means of Partial Least Squares (PLS) regression.

\section{Materials and Methods}

\subsection{Grated cheese samples}

Samples of grated Parmigiano Reggiano cheese containing varying rind percentages (RP) were provided by Parmigiano Reggiano cheese Consortium. More in detail, the grated cheese samples were prepared considering the following 15 percentages $(\mathrm{w} / \mathrm{w})$ of rind in pulp: $0 \%, 5 \%, 10 \%, 12 \%$ $14 \%, 16 \%, 18 \%, 20 \%, 22 \%, 24 \%, 26 \%, 28 \%, 30 \%, 35 \%$ and $40 \%$. 
In order to minimize possible effects of unwanted variations, the mixtures were prepared starting from the same matrices of cheese pulp and rind, obtained by grating pulp and rind pieces derived from different cheese wheels matured for a period of 12 months. The mixtures were replicated twice (deliveries A and B) as reported in Table 1, each time following a different random order. Firstly, the matrices of grated pulp and rind were prepared, and a part of them was then used to obtain the first set of 15 mixtures. The remaining part of the grated pulp and rind matrices was stored in the dark at $4{ }^{\circ} \mathrm{C}$, and after one week it was used to prepare the second set of 15 mixtures. For both the replicate sets, the samples were stored in the dark at $4^{\circ} \mathrm{C}$ and the day after their preparation they were delivered to the laboratory, where they were immediately analysed.

Furthermore, 15 additional samples with unknown rind percentage were provided by Parmigiano Reggiano cheese Consortium (X1-X15). These samples were prepared, delivered and stored considering the same procedure followed for the samples with known RP values.

\subsection{Image acquisition}

For each sample, three randomly sampled aliquots containing about $13 \mathrm{~g}$ of grated cheese were collected and placed inside a plastic Petri dish of $5.5 \mathrm{~cm}$ diameter. Each hyperspectral image included the three aliquots of two different samples. More in detail, the samples were positioned according to a $3 \times 2$ chessboard scheme, as reported in Figure 1 .

The hyperspectral images were acquired using a line scanning system (NIR Spectral Scanner, DV Optic) equipped with a Specim Imspector N17E imaging spectrometer coupled to a Xenics Xeva1.7-320 camera $(320 \times 256$ pixels $)$ embedding Specim Oles $31 \mathrm{f} / 2.0$ optical lens. The hyperspectral system covers the $900-1700 \mathrm{~nm}$ spectral range with a spectral resolution equal to $5 \mathrm{~nm}$. Due to the low signal-to-noise ratio at the edges of the spectral range, only 143 spectral channels between 960 and $1670 \mathrm{~nm}$ were considered for further analysis.

The hyperspectral images were acquired using a black silicon carbide sandpaper sheet as background and including in the image scene also a white ceramic tile with a $99 \%$ reflectance standard reference and two ceramic tiles with intermediate reflectance values corresponding to 89 $\%$ and $46 \%$, respectively. The raw data were converted into reflectance values using the instrumental calibration based on the measure of the white high reflectance standard reference and of the dark current (Ulrici et al., 2013).

\subsection{Image elaboration}

The first step of image elaboration consisted in the application of an additional internal calibration procedure in order to reduce possible variations over time. This correction procedure is based on the 
comparison of the average reflectance values of the white standard reference, of the two ceramic tiles and of the black silicon carbide sandpaper between an image chosen as reference and all the remainder images of the dataset. Further details about the image correction algorithm can be found in Ulrici et al. (2013).

Subsequently, the corrected images were cropped in order to obtain a single hyperspectral image for each aliquot of grated cheese sample. At the end of the cropping procedure, a total of 135 hyperspectral images were obtained ( $=45$ grated cheese samples $\times 3$ aliquots), as reported in the last column of Table 1.

After cropping, the pixels related to the black sandpaper background were removed using a thresholding procedure carried out considering a wavelength equal to $1100 \mathrm{~nm}$. Indeed, at $1100 \mathrm{~nm}$ the pixels with reflectance value lower than 0.5 were ascribable to the background or to the plastic Petri dish, thus they were not considered in the subsequent steps.

Finally, an additional morphological erosion procedure was performed using a disk-shaped structuring element with radius equal to 8 pixels (Van Den Boomgaard and Van Balen, 1992). Morphological erosion allowed to remove the pixels placed at the edges of the region of interest obtained after background removal, since these pixels were mainly influenced by scattering phenomena and specular reflections of the plastic Petri dish.

The image elaboration steps, which are summarized in Step 1 and Step 2 of Figure 1, were performed with routines written ad hoc in MATLAB language (ver. 9.3, The Mathworks Inc., USA).

\subsection{Data analysis}

\subsubsection{Exploratory analysis}

As a preliminary assessment, Principal Component Analysis (PCA) was performed at the pixel level on some representative images (Prats-Montalbán et al., 2011). More in detail, three images corresponding to RP values equal to $0 \%, 20 \%$ and $40 \%$ were merged together and analysed by means of PCA after applying standard normal variate (SNV) and mean center as spectral preprocessing methods. This preliminary analysis was carried out in order to obtain a qualitative evaluation of the differences between samples containing an increasing amount of rind.

\subsubsection{Calibration models}

\section{Data organization}

Before calculating the calibration model to predict the rind percentage, the hyperspectral images of the grated cheese samples were split into training images, for model computation, and test images 
for external validation. The training images included the hyperspectral images of grated cheese samples with RP values equal to $0 \%, 10 \%, 14 \%, 18 \%, 22 \%, 26 \%, 30 \%$ and $40 \%$, for a total of 48 images ( $=8 \mathrm{RP}$ values $\times 2$ deliveries $\times 3$ aliquots). The remainder images were separated into two different test sets:

- $\mathrm{TS}_{\mathrm{known}}$ : including the images acquired on cheese samples with $\mathrm{RP}$ values equal to 5\%, $12 \%$, $16 \%, 20 \%, 24 \%, 28 \%$ and $35 \%$, for a total of 42 images (= $7 \mathrm{RP}$ values $\times 2$ deliveries $\times 3$ aliquots);

- $\mathrm{TS}_{\text {unknown }}$ : including the images acquired on the cheese samples of unknown composition, for a total of 45 images (= 15 unknown samples $\times 3$ aliquots).

\section{Conversion into Common Space Hyperspectrograms}

The hyperspectral images were then converted into one-dimensional signals, named Common Space Hyperspectrograms (CSH) (Calvini et al., 2016). The basic idea behind the hyperspectrograms approach consists in converting each hyperspectral image of the dataset into a one-dimensional signal, which acts like a feature vector retaining the useful spectral/spatial information of the corresponding image (Ferrari et al., 2013; Ferrari et al., 2015). More in detail, in the case of CSH, the signals are obtained by merging in sequence the frequency distribution curves of quantities derived from a common PCA model, i.e. from a model calculated considering all the images of the training set.

The first step in the computation of the CSH consisted in unfolding all the three-dimensional hyperspectral images into two-dimensional matrices with as many rows as the pixels retained after background removal and erosion, and as many columns as the number of spectral channels. Then, the unfolded hypercubes were row-preprocessed using SNV and scaled according to the global mean spectrum, obtained by averaging all the retained pixel spectra of the training images. After unfolding and spectra preprocessing, for each training image the corresponding variance-covariance matrix was calculated. Then, all the resulting variance-covariance matrices were summed in order to obtain the kernel variance-covariance matrix of the whole training set (Geladi and Grahn, 1996). The kernel-variance covariance matrix was then decomposed by singular value decomposition (SVD) to obtain the loading vectors of the common PC space. In this case, the common PC space was calculated considering 3 principal components, based on the results of the previous exploratory data analysis described in Section 2.4.1.

Once calculated the PC space common to the training images, all the hyperspectral images belonging to both training and test sets were projected onto the PC space to obtain the corresponding scores, Q residuals and Hotelling's $\mathrm{T}^{2}$ vectors. Finally, for each image, the 
corresponding CSH signal was obtained by merging in sequence the frequency distribution curves of the three score vectors, of the Q residuals vector and of the Hotelling's $\mathrm{T}^{2}$ vector.

The frequency distribution curves were calculated considering a number of bins equal to 150 and normalized according to the number of pixels retained after image elaboration, as described in Section 2.3, to give the corresponding hyperspectrogram. Therefore, in this case each hyperspectrogram was a 750 points long vector, resulting from 150 bins $\times 5$ quantities derived from PCA (3 PCs + Q residuals + Hotelling's $\mathrm{T}^{2}$ ). Further details about the algorithm used to calculate the CSH can be found in Calvini et al. (2016). The conversion of the hyperspectral images into CSH signals is schematically depicted in Step 3 of Figure 1.

In this manner, at the end of the conversion procedure three different matrices of signals were obtained: the training set (TR), the test set derived from the $\mathrm{TS}_{\text {known }}$ images and the test set of unknown samples derived from the $\mathrm{TS}_{\text {unknown }}$ images.

Figure 2 shows a plot of the CSH signals belonging to the training set, coloured according to the rind percentage of the corresponding sample.

\section{Calibration model}

The training set matrix containing the $\mathrm{CSH}$ signals calculated from the training images was used to calculate the calibration model to predict the RP value, using Partial Least Squares (PLS) algorithm (Geladi and Kowalski, 1986). The signals were preprocessed using mean center and the optimal number of latent variables (LVs) was chosen by minimizing the cross-validation error. In particular, a custom cross-validation scheme was followed, considering 2 deletion groups, each one containing the signals derived from samples belonging to the same delivery day.

The performances of the calibration models were evaluated both in terms of Root-Mean-Square Error (RMSE) and of coefficient of determination $\left(\mathrm{R}^{2}\right)$. These parameters were calculated in calibration (RMSEC and $\mathrm{R}^{2} \mathrm{Cal}$ ), cross-validation (RMSECV and $\mathrm{R}^{2} \mathrm{CV}$ ) and prediction of the external test set (RMSEP and $\mathrm{R}^{2}$ Pred).

The conversion of the hyperspectral images in CSH signals was done using a specific Graphical User Interface (GUI), that was previously developed by some of the authors of the present work. The GUI, which works under the MATLAB environment (ver. 9.3, The Mathworks, USA) and is named Hyperspectrograms GUI, is freely downloadable from www.chimslab.unimore.it/downloads. PCA and PLS models were calculated using PLS Toolbox (ver. 8.5, Eigenvector Research Inc., USA) and MIA Toolbox (ver. 3.0.4, Eigenvector Research Inc., USA). 


\section{Results and discussion}

\subsection{PCA at the pixel-level}

For a first evaluation of the differences between grated cheese samples containing different amounts of rind, three hyperspectral images corresponding to samples with RP values equal to $0 \%, 20 \%$ and $40 \%$ were merged together to obtain a unique hyperspectral image, which was analysed at the pixellevel by PCA. Figure 3.a reports the resulting PC1-PC2 score plot, where each object represents a single pixel and is coloured according to pixel density, i.e. a yellowish colour represents a region of the PC1-PC2 score space with a high density of pixels, while blue corresponds to low pixel density. From this score plot it is possible to observe the presence of three clusters of pixels, corresponding to the imaged samples with different RP values. The separation between samples with different rind levels is particularly evident along PC2. Indeed, the sample containing only cheese pulp is characterized by higher PC2 score values, while samples with increasing percentages of rind have decreasing PC2 score values, as shown in the PC2 score image reported in Figure 3.b.

In order to investigate the spectral features involved in the definition of the PC space, the corresponding PC1-PC2 loading vectors are reported in Figure 3.c. The highest absolute values of the PC2 loading vector can be found in the 1195-1225 nm wavelength range, corresponding to the C-H bond second overtone ascribable to lipids (Burns and Ciurzak, 2008; Karoui et al., 2006), in the 1330-1340 $\mathrm{nm}$ spectral range corresponding to asymmetric stretching vibration of water (Ozaky, 2002), and in the region centred at $1400 \mathrm{~nm}$ ascribable to the $\mathrm{O}-\mathrm{H}$ bond first overtone of free water (Burns and Ciurzak, 2008).

Therefore, the amount of rind of the cheese samples can be somehow described by the distribution of the corresponding pixel spectra along the principal components. A convenient way to summarize this pixel distribution consists in using the frequency distribution curves of the score vectors of each sample, as reported in Figure 3.a that shows the frequency distribution curves of both PC1 and PC2 score vectors for each image. From this figure it is possible to observe the presence of a clear shift of the frequency distribution curves of $\mathrm{PC} 2$, which is related to the rind percentage of the corresponding samples. Although less marked, a variation with RP can be observed also for the frequency distribution curves of PC1 scores, which tend to become sharper and with a maximum located at lower PC1 values with increasing values of rind percentage.

Since the hyperspectrograms approach is based on the use of frequency distribution curves of score vectors calculated from a PCA model in order to summarize the relevant information contained in the images, this preliminary analysis suggests the effectiveness of this approach for the determination of the rind amount in hyperspectral images of grated cheese samples. 


\subsection{PLS calibration model with the CSH approach}

The training set of CSH signals reported in Figure 2 was then used to calculate a PLS regression model for the quantification of rind percentage in the samples of grated Parmigiano Reggiano cheese, leading to the results reported in Table 2.

The optimal model dimensionality was found to be equal to $8 \mathrm{LVs}$, leading to a RMSECV value equal to $1.70 \mathrm{RP}$ units, corresponding to a $\mathrm{R}^{2} \mathrm{CV}$ value of 0.979 .

The calibration model was then used to predict the RP of the samples belonging to the $\mathrm{TS}_{\text {known }}$ test set. In this case, the prediction results were calculated considering both the whole range of rind levels $(0 \%-40 \%)$ and only the interval of rind percentages ranging from $10 \%$ to $30 \%$, which better reflects RP values that generally may occur in real situations.

The prediction results confirm the effectiveness of the calibration model in quantifying the rind percentage, leading to RMSEP values equal to 1.91 and 1.85 RP units in the $0-40 \%$ and $10-30 \%$ ranges, respectively.

Figure 4 shows the plot of the rind percentage values predicted for the $\mathrm{TS}_{\text {known }}$ test set versus the corresponding experimental values, where the samples are coloured according to the delivery day. Generally, all the objects are close to the bisector, indicating the good prediction performances of the model. In addition, from Figure 4 it is also possible to observe that there are not evident systematic variations in the prediction results caused by the different delivery days, which further confirms the robustness of the calibration model toward replicated measurements.

Considering that compliant Parmigiano Reggiano grated cheese samples should have an RP value lower or equal than $18 \%$, all the test set samples with a rind percentage falling outside this limit are correctly identified by the model.

The calibration model was also used to predict the samples with unknown composition belonging to the $\mathrm{TS}_{\text {unknown }}$ test set. The predicted rind percentages of the unknown samples were communicated to the Parmigiano Reggiano Cheese Consortium, which then revealed the corresponding experimental values. In this manner, it was possible to perform a further external validation of the calibration model.

It has to be considered that, for each unknown sample, three different aliquots were imaged and, therefore, three RP values were obtained in prediction by the model. In order to have a single estimate of the RP value for each unknown sample, the three RP predicted values corresponding to the three aliquots were averaged for each sample. The results are reported in Table 3, together with the corresponding experimental values, further confirming the good prediction ability of the calibration model. More in detail, an RMSEP value equal to $2.50 \mathrm{RP}$ units was obtained, corresponding to an $\mathrm{R}^{2}$ value equal to 0.955 . The highest difference between predicted and 
experimental rind percentage, equal to $5 \mathrm{RP}$ units, was observed for sample $\mathrm{X} 15$, while samples X2 and $\mathrm{X} 4$ were exactly predicted.

In order to have a comprehensive evaluation of the hyperspectrogram regions most relevant to the calibration model, Figure 5.a reports the Variable Importance in Projection (VIP) scores: variables with VIP score values higher than one are considered significant for the model. This figure shows that all the frequency distribution curves of the PCA quantities included in the CSH signals have regions with significant variables. In particular, among the three score vectors included in the signals, the regions related to the frequency distribution curve of PC2 reach the highest VIP score values, together with the signal regions related to Hotelling $\mathrm{T}^{2}$ vales.

As an example, Figure 5.b reports the PC2 score images of some representative test set samples with increasing percentage of rind. Similarly to what was previously reported in Section 3.1, images of samples with a lower RP value are characterized by higher PC2 score values, and the increase of the RP value in the grated cheese samples causes a shift of the corresponding pixels toward lower PC2 score values. Actually, this is due to the fact that the PC2 loading vector of the common PCA model used to calculate the CSH signals is very similar to the PC2 loading vector calculated on the three sample hyperspectral images, reported in Figure 3c.

\section{Conclusions}

The present study demonstrated the possibility of using NIR-HSI as a tool for the quantification of the amount of rind in grated Parmigiano Reggiano cheese samples. The combined use of a data dimensionality reduction approach, namely the Common Space Hyperspectrograms approach, with PLS regression allowed to obtain satisfactory prediction performances. The calibration model was validated using two different test sets: the first test set consisted of cheese samples with known RP values, while for the second test set the experimental RP values of the analysed samples were revealed by the operators of Parmigiano Reggiano Cheese Consortium only after providing them with the RP values predicted by the model. The RMSEP values obtained for both test sets, corresponding to 1.91 RP units and 2.50 RP units, respectively, confirm the advantages of coupling spatial and spectral information of a sample brought by NIR-HSI in the analysis of heterogeneous products, like grated cheese.

However, it has to be considered that commercial samples of grated Parmigiano Reggiano cheese may be affected by different variability factors, such as months of ripening, fat content and rind processing methods, among others. Therefore, in order to obtain a more robust calibration model the influence of these factors should be properly evaluated and included in the model. As a 
373 consequence, the increasing complexity of the model should be faced by including further steps in 374 the data analysis workflow, like e.g. spectral and/or spatial feature selection.

375

376 Acknowledgements

377 The authors wish to thank Consorzio del Formaggio Parmigiano Reggiano for providing technical 378 and financial support.

379 


\section{References}

Alinovi, M., Mucchetti, G., \& Tidona, F. (2019). Application of NIR spectroscopy and image analysis for the characterisation of grated Parmigiano-Reggiano cheese. International dairy journal, 92, 50-58.

Amigo, J. M., Martí, I., \& Gowen, A. (2013). Hyperspectral imaging and chemometrics: a perfect combination for the analysis of food structure, composition and quality. In Data handling in science and technology (Vol. 28, pp. 343-370). Elsevier.

Amigo, J. M., Babamoradi, H., \& Elcoroaristizabal, S. (2015). Hyperspectral image analysis. A tutorial. Analytica chimica acta, 896, 34-51.

Burger, J., \& Geladi, P. (2006). Hyperspectral NIR imaging for calibration and prediction: a comparison between image and spectrometer data for studying organic and biological samples. Analyst, 131(10), 1152-1160.

Burger, J., \& Gowen, A. (2011). Data handling in hyperspectral image analysis. Chemometrics and Intelligent Laboratory Systems, 108(1), 13-22.

Burns, D. A., Ciurczak, E. W. (2008) Handbook of near infrared analysis, CRC Press, Boca Raton, Florida, USA, (Chapter 17, pp. 347-386). ISBN 978-0-8493-7393-0

Calvini, R., Foca, G., \& Ulrici, A. (2016). Data dimensionality reduction and data fusion for fast characterization of green coffee samples using hyperspectral sensors. Analytical and bioanalytical chemistry, 408(26), 7351-7366.

Calvini, R., Orlandi, G., Foca, G., Ulrici, A. (2018). Development of a classification algorithm for efficient handling of multiple classes in sorting systems based on hyperspectral imaging. Journal of Spectral Imaging, 7, a13.

Cattaneo, S., Hogenboom, J. A., Masotti, F., Rosi, V., Pellegrino, L., \& Resmini, P. (2008). Grated Grana Padano cheese: new hints on how to control quality and recognize imitations. Dairy Science and Technology, 88(4-5), 595-605.

Cevoli, C., Gori, A., Nocetti, M., Cuibus, L., Caboni, M. F., \& Fabbri, A. (2013). FT-NIR and FTMIR spectroscopy to discriminate competitors, non compliance and compliance grated Parmigiano Reggiano cheese. Food research international, 52(1), 214-220.

Cevoli, C., Fabbri, A., Gori, A., Caboni, M. F., \& Guarnieri, A. (2013). Screening of grated cheese authenticity by nir spectroscopy. Journal of Agricultural Engineering, 44, 264-267.

Corti, M., Gallina, P. M., Cavalli, D., \& Cabassi, G. (2017). Hyperspectral imaging of spinach canopy under combined water and nitrogen stress to estimate biomass, water, and nitrogen content. Biosystems engineering, 158, 38-50.

Čurda, L., \& Kukačková, O. (2004). NIR spectroscopy: a useful tool for rapid monitoring of processed cheeses manufacture. Journal of Food Engineering, 61(4), 557-560.

Dale, L. M., Thewis, A., Boudry, C., Rotar, I., Dardenne, P., Baeten, V., \& Pierna, J. A. F. (2013). Hyperspectral imaging applications in agriculture and agro-food product quality and safety control: a review. Applied Spectroscopy Reviews, 48(2), 142-159.

Ferrari, C., Foca, G., \& Ulrici, A. (2013). Handling large datasets of hyperspectral images: Reducing data size without loss of useful information. Analytica chimica acta, 802, 29-39.

Ferrari, C., Foca, G., Calvini, R., \& Ulrici, A. (2015). Fast exploration and classification of large hyperspectral image datasets for early bruise detection on apples. Chemometrics and Intelligent Laboratory Systems, 146, 108-119. 
Foca, G., Salvo, D., Cino, A., Ferrari, C., Fiego, D. P. L., Minelli, G., \& Ulrici, A. (2013). Classification of pig fat samples from different subcutaneous layers by means of fast and nondestructive analytical techniques. Food research international, 52(1), 185-197.

Geladi, P., \& Grahn, H. F. (1996). Multivariate image analysis. Encyclopedia of Analytical Chemistry.

Geladi, P., \& Kowalski, B. R. (1986). Partial least-squares regression: a tutorial. Analytica chimica acta, 185, 1-17.

Giraudo, A., Calvini, R., Orlandi, G., Ulrici, A., Geobaldo, F., Savorani, F. (2018). Development of an automated method for the identification of defective hazelnuts based on RGB image analysis and colourgrams. Food Control, 94, 233-240.

Gowen, A. A., O'Donnell, C., Cullen, P. J., Downey, G., \& Frias, J. M. (2007). Hyperspectral imaging-an emerging process analytical tool for food quality and safety control. Trends in food science \& technology, 18(12), 590-598.

Karoui, R., Mouazen, A. M., Dufour, É., Pillonel, L., Schaller, E., De Baerdemaeker, J., \& Bosset, J. O. (2006). Chemical characterisation of European Emmental cheeses by near infrared spectroscopy using chemometric tools. International Dairy Journal, 16(10), 1211-1217.

Karoui, R., Dufour, É., \& De Baerdemaeker, J. (2007). Front face fluorescence spectroscopy coupled with chemometric tools for monitoring the oxidation of semi-hard cheeses throughout ripening. Food Chemistry, 101(3), 1305-1314.

Kraggerud, H., Næs, T., \& Abrahamsen, R. K. (2014). Prediction of sensory quality of cheese during ripening from chemical and spectroscopy measurements. International Dairy Journal, 34(1), 6-18.

Kucheryavskiy, S. (2013). A new approach for discrimination of objects on hyperspectral images. Chemometrics and Intelligent Laboratory Systems, 120, 126-135.

Liu, Y., Pu, H., \& Sun, D. W. (2017). Hyperspectral imaging technique for evaluating food quality and safety during various processes: A review of recent applications. Trends in food science \& technology, 69, 25-35.

Malacarne, M., Summer, A., Formaggioni, P., Franceschi, P., Sandri, S., Pecorari, M., ... \& Mariani, P. (2008). Dairy maturation of milk used in the manufacture of Parmigiano-Reggiano cheese: effects on physico-chemical characteristics, rennet-coagulation aptitude and rheological properties. Journal of dairy research, 75(2), 218-224.

Malacarne, M., Summer, A., Franceschi, P., Formaggioni, P., Pecorari, M., Panari, G., \& Mariani, P. (2009). Free fatty acid profile of Parmigiano-Reggiano cheese throughout ripening: Comparison between the inner and outer regions of the wheel. International dairy journal, 19(10), 637-641.

Oliveri, P., Malegori, C., Casale, M., Tartacca, E., \& Salvatori, G. (2019). An innovative multivariate strategy for HSI-NIR images to automatically detect defects in green coffee. Talanta, 199, 270-276.

Orlandi, G., Calvini, R., Foca, G., Ulrici, A. (2018). Automated quantification of defective maize kernels by means of Multivariate Image Analysis. Food Control, 85, 259-268.

Orlandi, G., Calvini, R., Pigani, L., Foca, G., Vasile Simone, G., Antonelli, A., Ulrici, A. (2018). Electronic eye for the prediction of parameters related to grape ripening. Talanta, 186, 381-388.

Ozaki Y. (2002) Two dimensional near-infrared correlation spectroscopy. In: Siesler H.W., Ozaki Y., Kawata S. and Heise M. (Eds). Applications in chemistry in near-infrared spectroscopy: principles, instruments and applications, Wiley-VCH, Weinheim, Germany. 
Prats-Montalbán, J. M., De Juan, A., \& Ferrer, A. (2011). Multivariate image analysis: a review with applications. Chemometrics and intelligent laboratory systems, 107(1), 1-23.

Schönbichler, S. A., Bittner, L. K. H., Weiss, A. K. H., Griesser, U. J., Pallua, J. D., \& Huck, C. W. (2013). Comparison of NIR chemical imaging with conventional NIR, Raman and ATR-IR spectroscopy for quantification of furosemide crystal polymorphs in ternary powder mixtures. European Journal of Pharmaceutics and Biopharmaceutics, 84(3), 616-625.

Specifications of Parmigiano Reggiano Cheese. https://www.parmigianoreggiano.com/consortium/rules_regulation_2/default.aspx. Accessed 2 September 2019.

Ulrici, A., Foca, G., Ielo, M. C., Volpelli, L. A., \& Fiego, D. P. L. (2012). Automated identification and visualization of food defects using RGB imaging: Application to the detection of red skin defect of raw hams. Innovative Food Science \& Emerging Technologies, 16, 417-426.

Ulrici, A., Serranti, S., Ferrari, C., Cesare, D., Foca, G., \& Bonifazi, G. (2013). Efficient chemometric strategies for PET-PLA discrimination in recycling plants using hyperspectral imaging. Chemometrics and Intelligent Laboratory Systems, 122, 31-39.

Van Den Boomgaard, R., \& Van Balen, R. (1992). Methods for fast morphological image transforms using bitmapped binary images. CVGIP: Graphical Models and Image Processing, 54(3), 252-258.

Woodcock, T., Fagan, C. C., O’Donnell, C. P., \& Downey, G. (2008). Application of near and midinfrared spectroscopy to determine cheese quality and authenticity. Food and Bioprocess Technology, 1(2), 117-129.

Wu, D., \& Sun, D. W. (2013). Advanced applications of hyperspectral imaging technology for food quality and safety analysis and assessment: A review-Part I: Fundamentals. Innovative Food Science \& Emerging Technologies, 19, 1-14.

Wu, D., \& Sun, D. W. (2013). Advanced applications of hyperspectral imaging technology for food quality and safety analysis and assessment: A review-Part II: Applications. Innovative Food Science \& Emerging Technologies, 19, 15-28.

$\mathrm{Xu}$, J. L., Riccioli, C., \& Sun, D. W. (2016). Efficient integration of particle analysis in hyperspectral imaging for rapid assessment of oxidative degradation in salmon fillet. Journal of Food Engineering, 169, 259-271.

Zannoni, M., \& Hunter, E. A. (2015). Relationship between sensory results and compliance scores in grated Parmigiano-Reggiano cheese. Italian Journal of Food Science, 27(4), 487-494. 


\section{Captions to Tables and Figures}

501 Table 1. Summary information about the grated cheese samples considered in the present study

502 Table 2. Results of PLS regression for the determination of rind percentage.

503 Table 3. Prediction results of the unknown test samples and corresponding experimental RP values.

504

505 Figure 1. Key steps involved in image elaboration and analysis

506 Figure 2. Hyperspectrograms of the training set images; the signals are coloured according to the 507 rind percentage of the corresponding grated cheese sample

508 Figure 3. In (a) PC1-PC2 score plot of the image containing grated cheese sample with 0\%, 20\% 509 and $40 \%$ percentages of rind, and corresponding frequency distribution curves of PC1 and PC2 510 score vectors calculated separately for each sample. In (b) PC2 score image. In (c) PC1 and PC2 511 loading vectors.

512 Figure 4. Results of the PLS model: TSknown test set predicted rind percentage (Y Predicted) vs 513 experimental rind percentage (Y measured).

514 Figure 5. VIP scores of the PLS model (a) and PC2 score images of samples with increasing rind 515 percentage values(b). 


\section{Highlights}

- Rind percentage in grated Parmigiano Reggiano cheese should not exceed $18 \%$ (w/w)

- Hyperspectral imaging (HSI) was used to estimate rind percentage in grated cheese

- The images were converted in one-dimensional signals named hyperspectrograms

- The hyperspectrograms were used to build PLS calibration models

- Validation with test samples confirmed the effectiveness of the proposed approach 
${ }^{*}$ Conflict of Interest Form

\section{Conflict of interest}

The authors declare that they have no conflict of interest. 
1. Image correction

1. and cropping
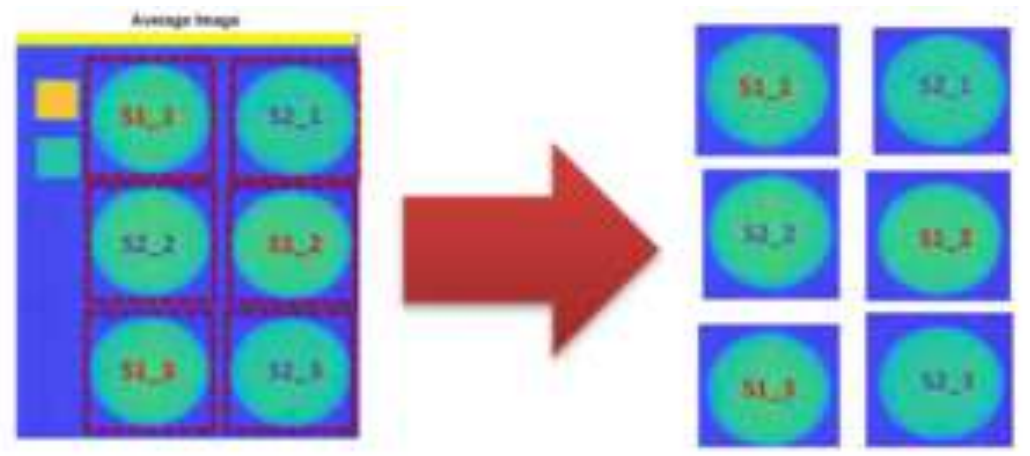

2. Background removal

2. and erosion
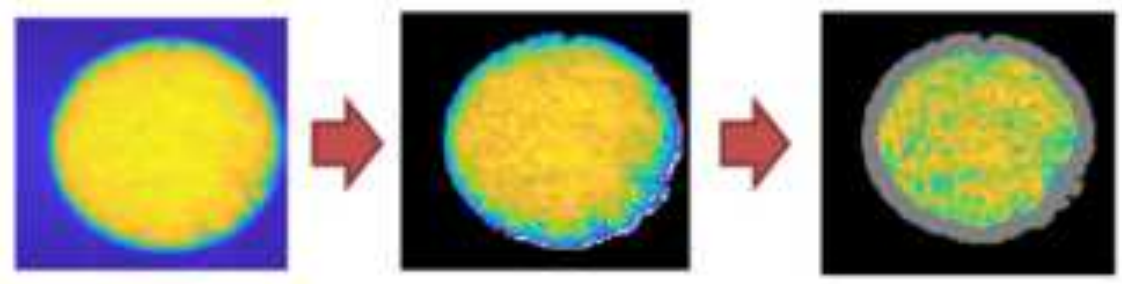

TR images

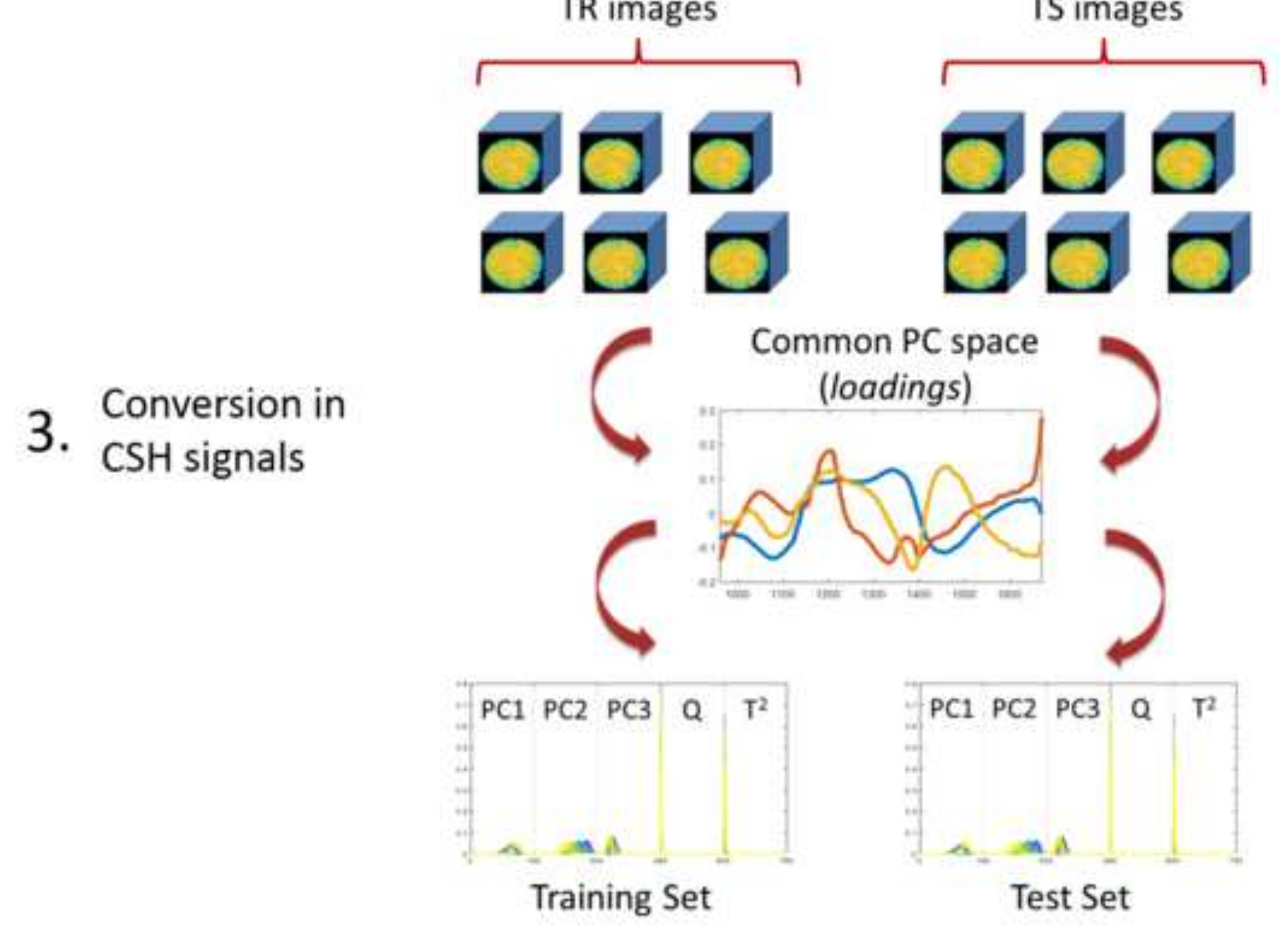

TS images

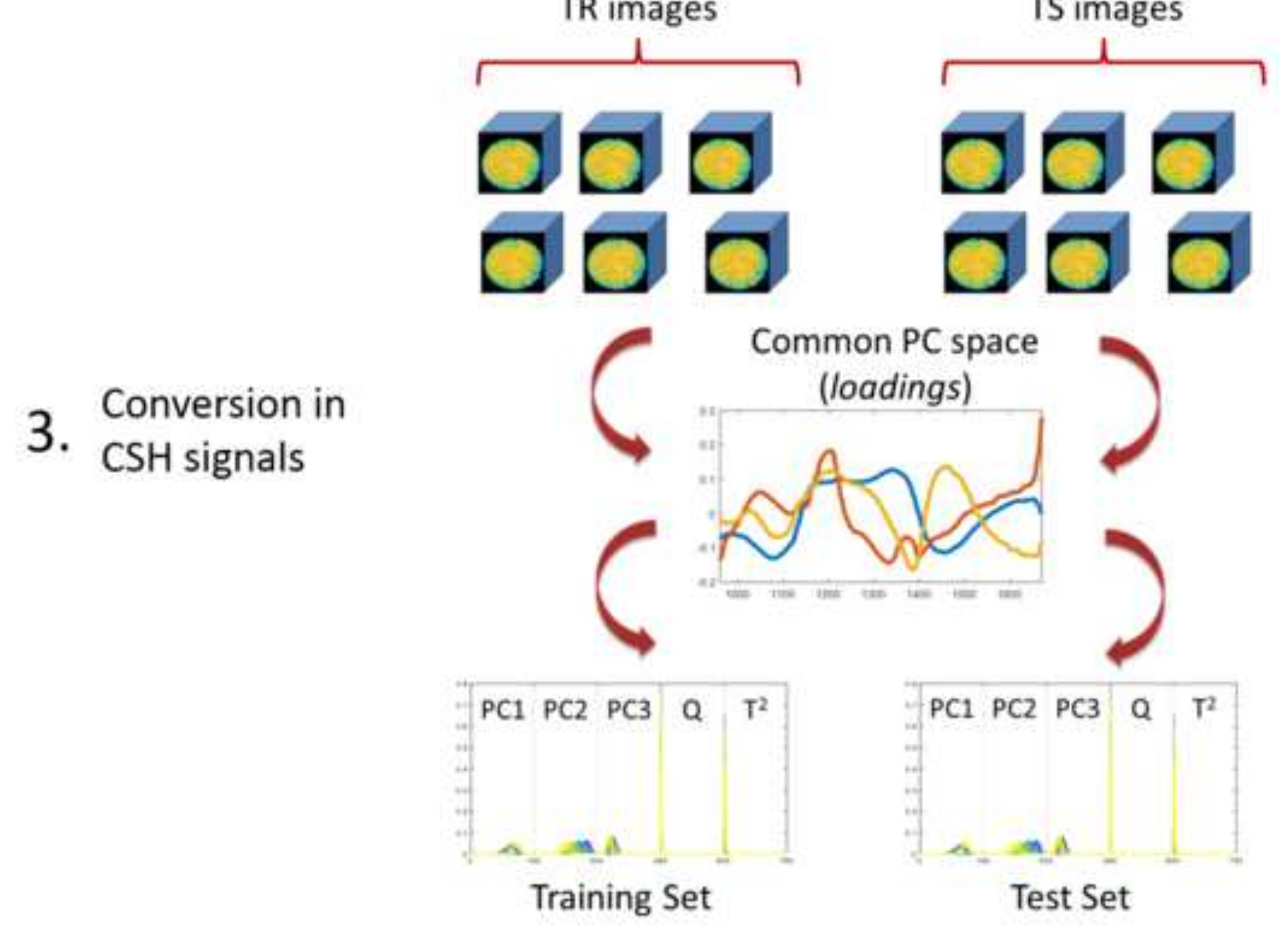

Common PC space

3. Conversion in

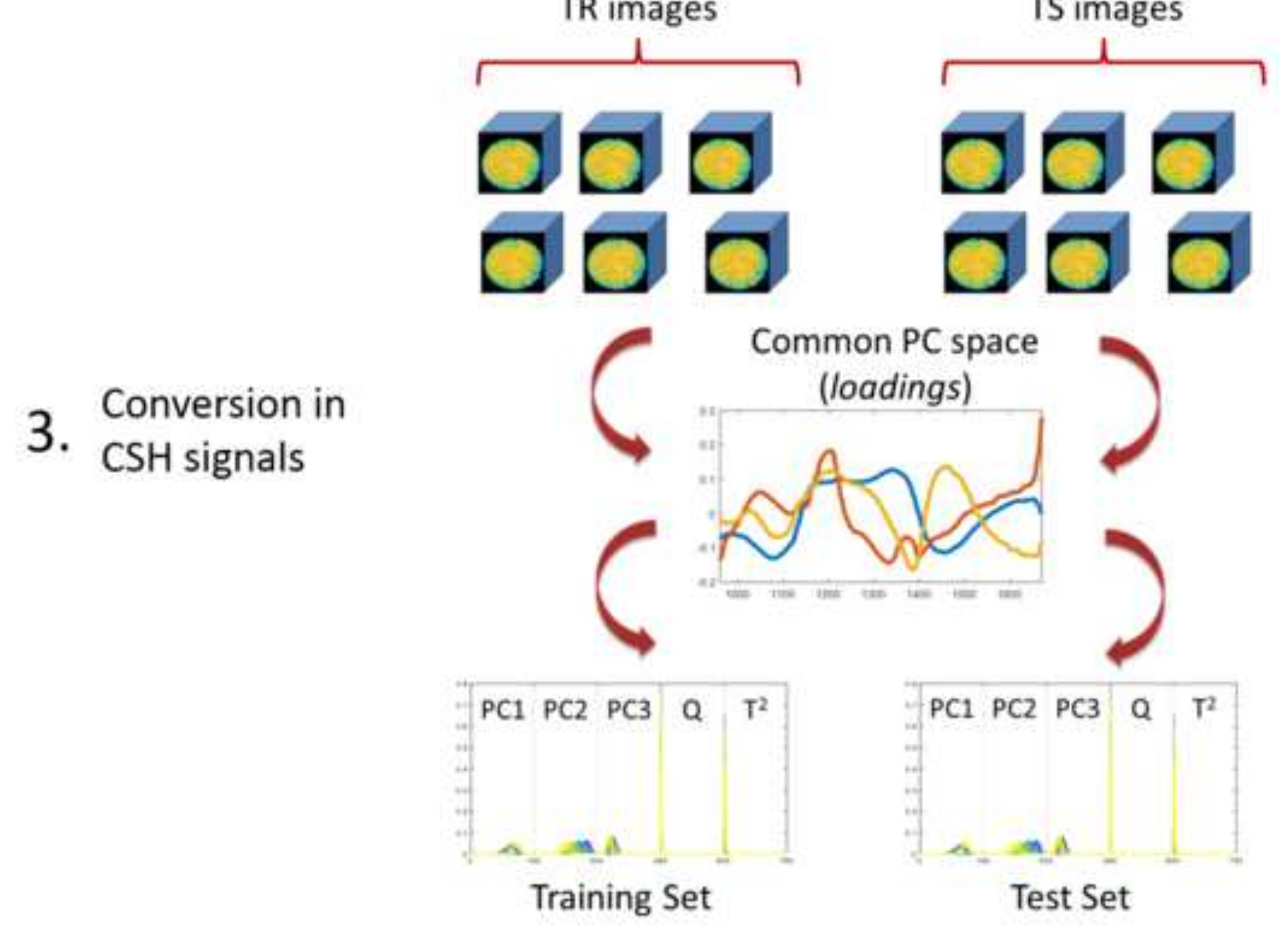




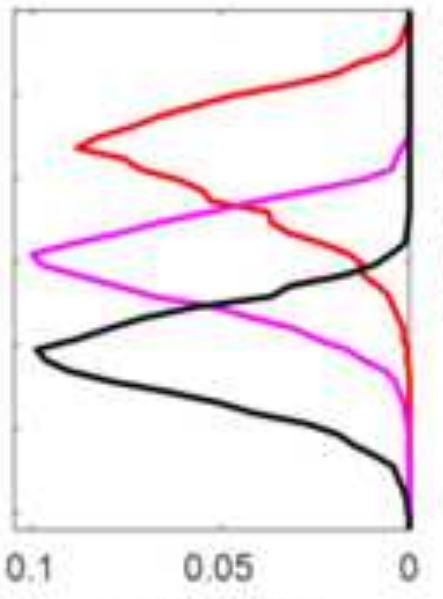

Frequency

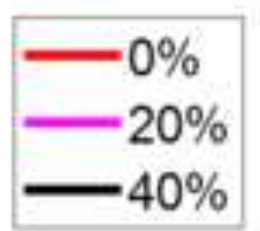

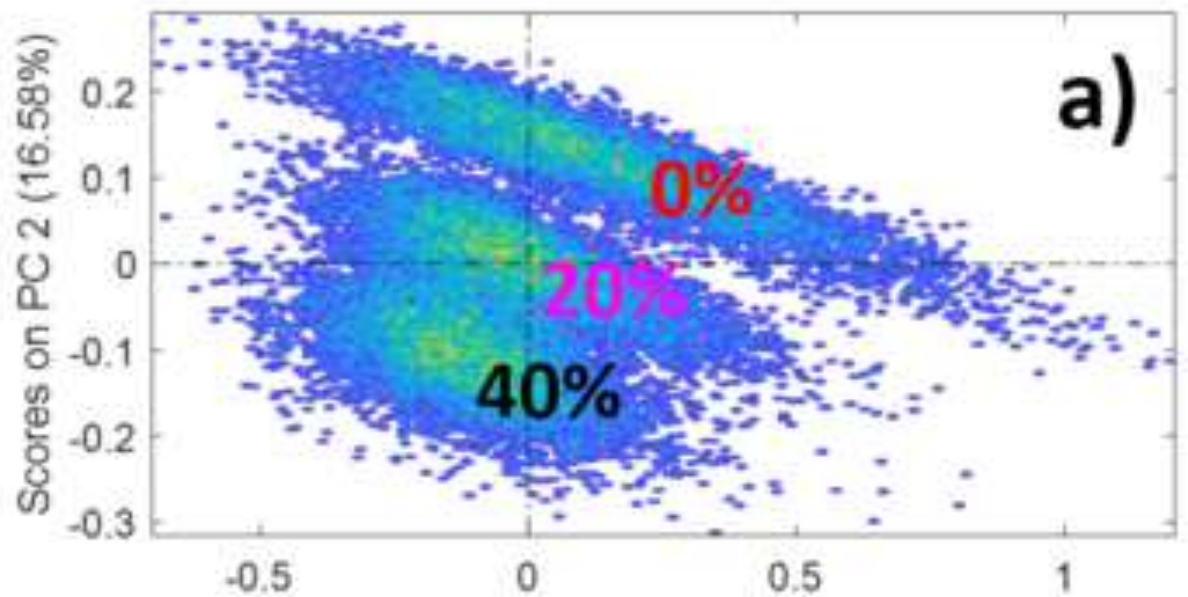

Scores on PC 1 (72.83\%)

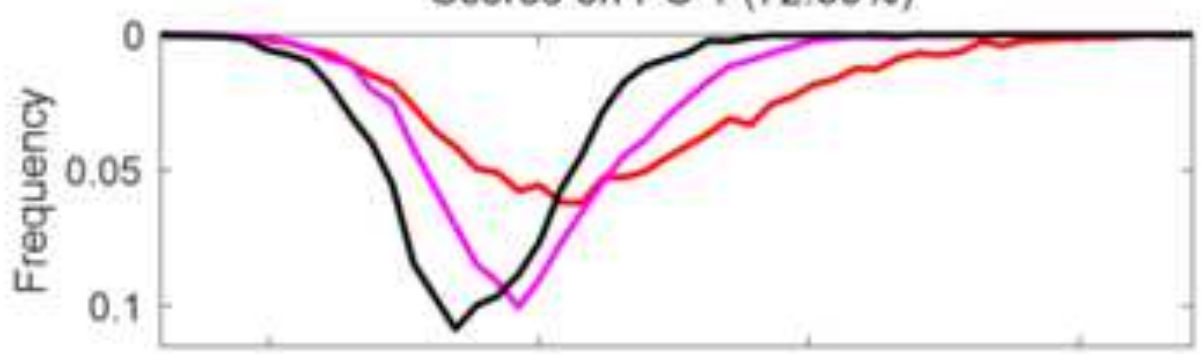

Image of Scores on PC 2 (16.58\%)

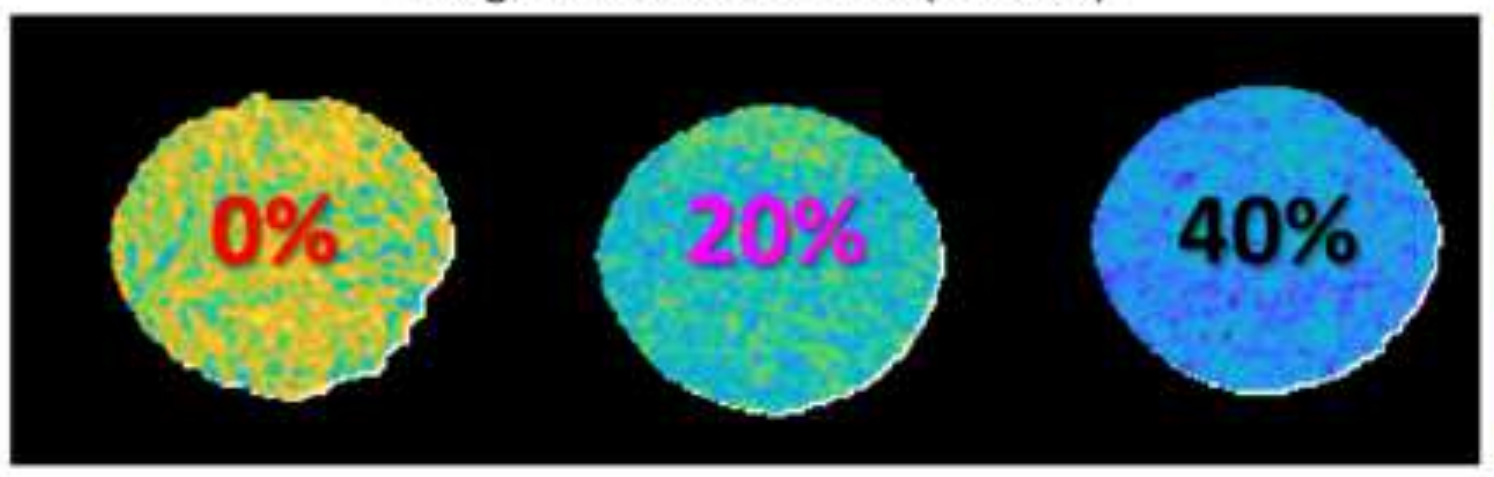

b)
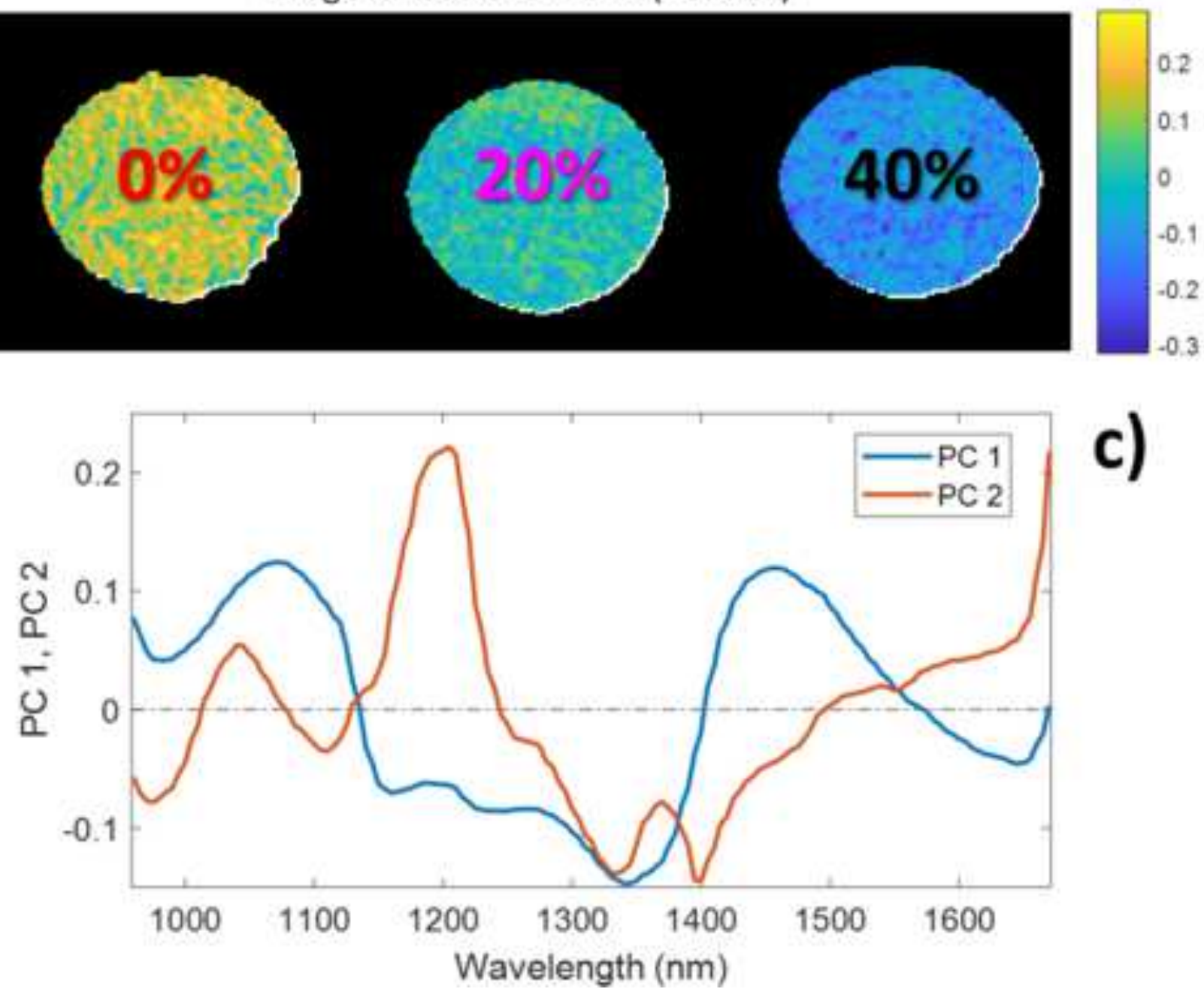


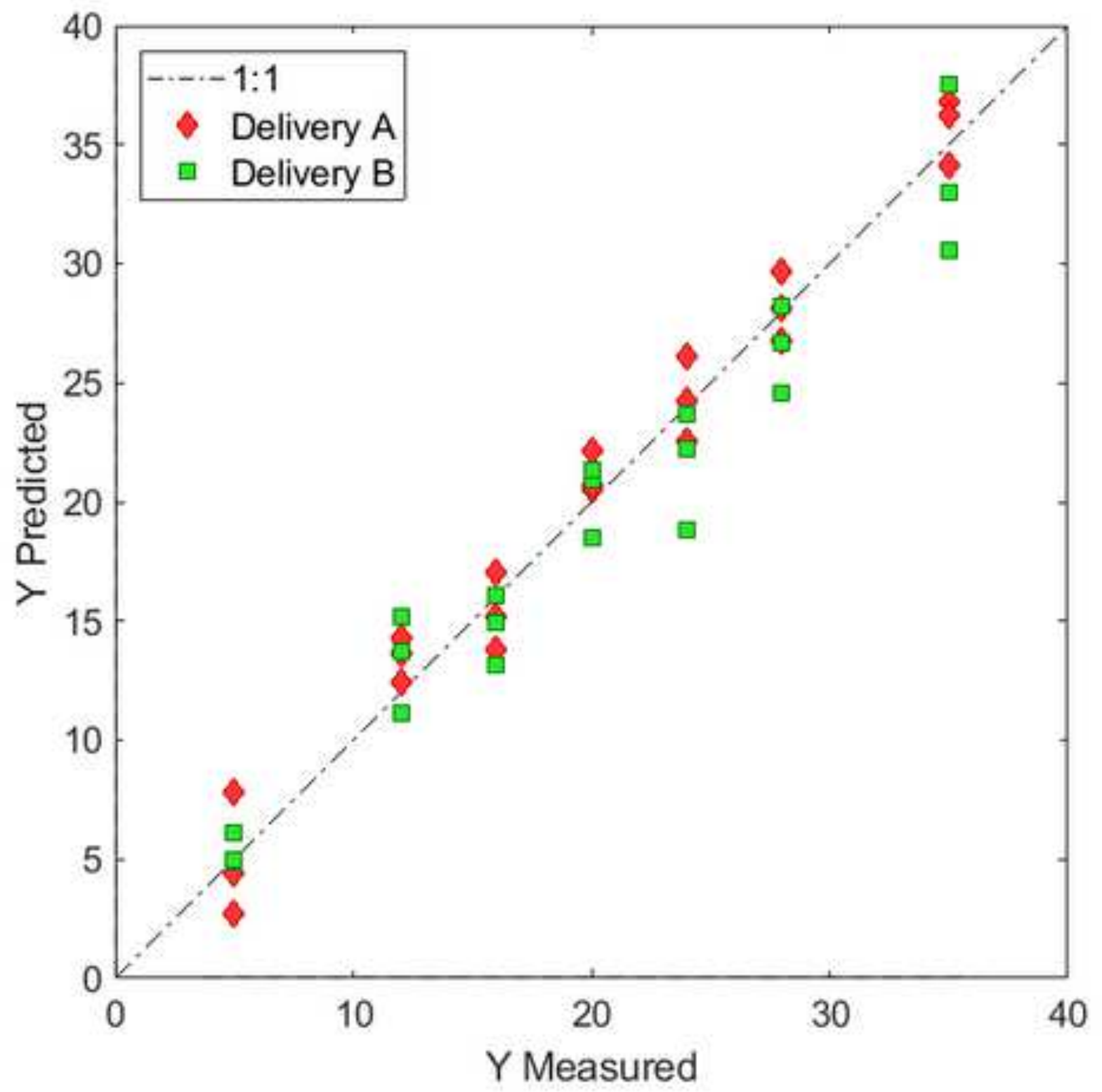



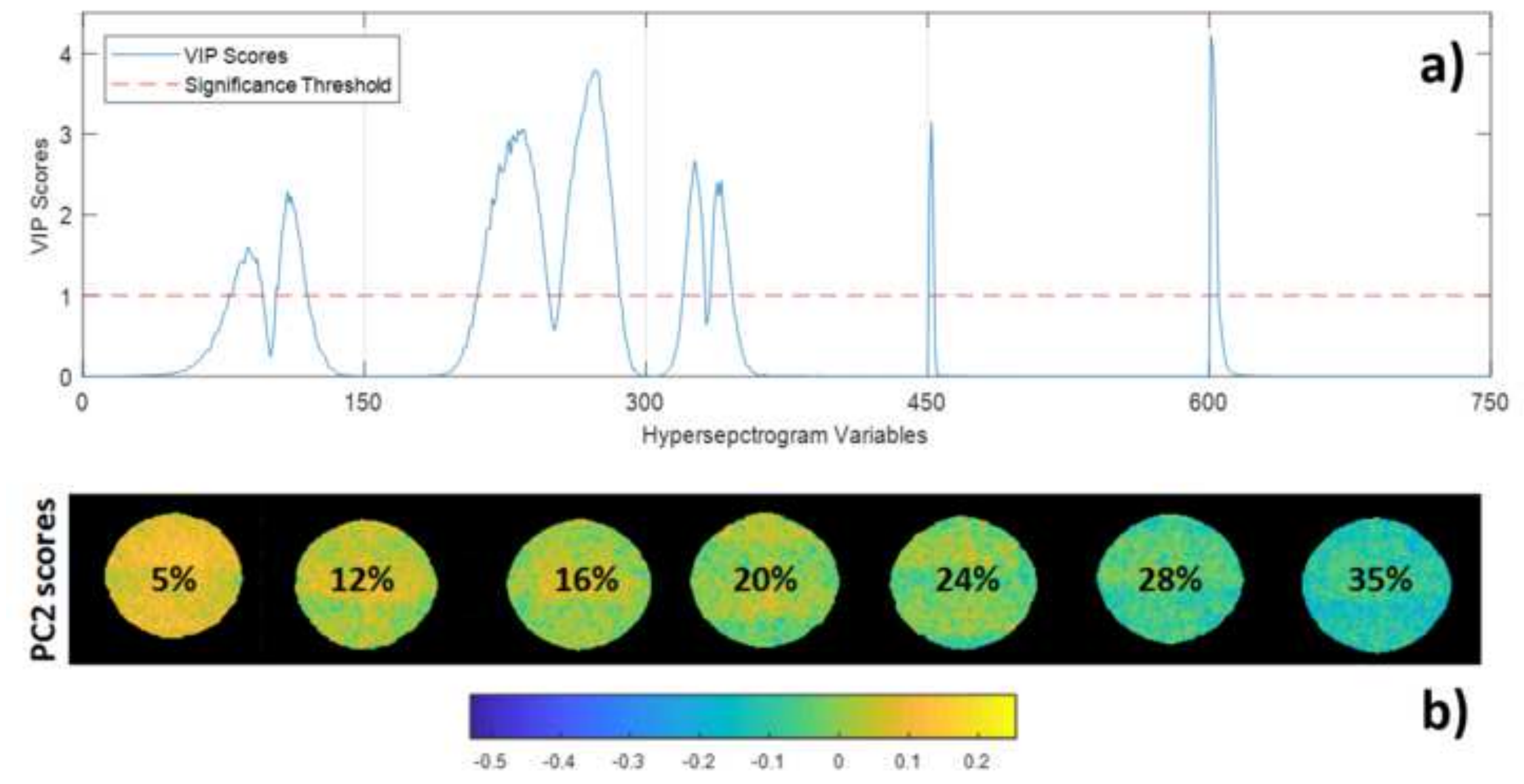
Table 1. Summary information about the grated cheese samples considered in the present study

\begin{tabular}{|c|c|c|c|c|}
\hline & $\begin{array}{c}\text { Delivery } \\
\text { Date }\end{array}$ & $\mathbf{R P}(\mathbf{w} / \mathbf{w})$ & $\begin{array}{c}\text { Number of } \\
\text { samples }\end{array}$ & $\begin{array}{c}\text { Number of } \\
\text { images }\end{array}$ \\
\hline \multirow[t]{2}{*}{$\begin{array}{c}\text { Delivery } \\
\text { A }\end{array}$} & \multirow[t]{2}{*}{$12 / 04 / 2018$} & $\begin{array}{c}0 \%, 5 \%, 10 \%, 12 \%, 14 \%, 16 \%, \\
18 \%, 20 \%, 22 \%, 24 \%, 26 \%, 28 \%, \\
30 \%, 35 \%, 40 \%\end{array}$ & 15 & $45(=15 \times 3)$ \\
\hline & & $\mathrm{X} 1-\mathrm{X} 7$ & 7 & $21(=7 \times 3)$ \\
\hline \multirow[t]{2}{*}{$\begin{array}{c}\text { Delivery } \\
\text { B }\end{array}$} & \multirow[t]{2}{*}{$12 / 11 / 2018$} & $\begin{array}{c}0 \%, 5 \%, 10 \%, 12 \%, 14 \%, 16 \% \\
18 \%, 20 \%, 22 \%, 24 \%, 26 \%, 28 \%, \\
30 \%, 35 \%, 40 \%\end{array}$ & 15 & $45(=15 \times 3)$ \\
\hline & & X8-X15 & 8 & $24(=8 \times 3)$ \\
\hline
\end{tabular}


Table 2. Results of PLS regression for the determination of rind percentage.

\begin{tabular}{|c|c|}
\hline LVs & 8 \\
\hline RMSEC & 0.98 \\
\hline RMSECV & 1.70 \\
\hline RMSEP $_{\mathbf{0 - 4 0} \%}$ & 1.91 \\
\hline RMSEP $_{\mathbf{1 0 - 3 0} \%}$ & 1.85 \\
\hline $\mathbf{R}^{\mathbf{2}}$ Cal & 0.993 \\
\hline $\mathbf{R}^{\mathbf{2}}$ CV & 0.979 \\
\hline $\mathbf{R}^{\mathbf{2}}$ Pred $_{\mathbf{0 - 4 0} \%}$ & 0.958 \\
\hline $\mathbf{R}^{\mathbf{2}}$ Pred $_{\mathbf{1 0 - 3 0} \%}$ & 0.893 \\
\hline
\end{tabular}


Table 3. Prediction results of the unknown test samples and corresponding experimental RP values.

\begin{tabular}{|c|c|c|}
\hline $\begin{array}{c}\text { Sample } \\
\text { name }\end{array}$ & $\begin{array}{c}\text { Predicted } \\
\text { RP }\end{array}$ & $\begin{array}{c}\text { Experimental } \\
\text { RP }\end{array}$ \\
\hline X1 & 30 & 32 \\
\hline $\mathrm{X} 2$ & 23 & 23 \\
\hline X3 & 20 & 18 \\
\hline $\mathrm{X4}$ & 0 & 0 \\
\hline X5 & 7 & 5 \\
\hline X6 & 24 & 23 \\
\hline X7 & 13 & 10 \\
\hline X8 & 43 & 40 \\
\hline X9 & 14 & 12 \\
\hline X10 & 18 & 16 \\
\hline X11 & 30 & 28 \\
\hline X12 & 9 & 5 \\
\hline $\mathrm{X} 13$ & 35 & 32 \\
\hline X14 & 1 & 0 \\
\hline X15 & 23 & 18 \\
\hline
\end{tabular}

